\title{
First dust grains from outside the Solar System
}

Citizen-science project identifies particles caught by NASA craft as having likely interstellar origin.

\section{Nicole Skinner}

14 August 2014

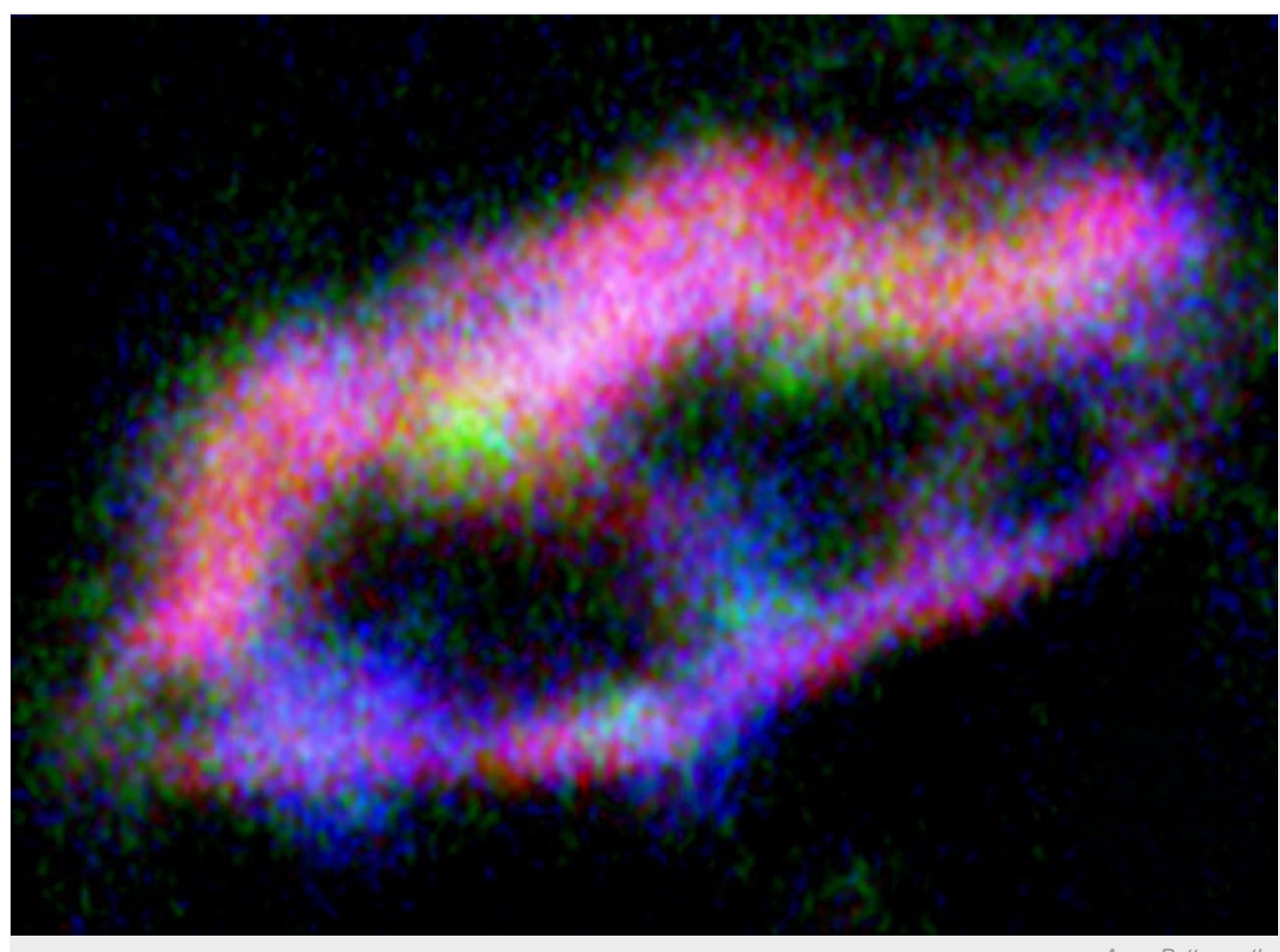

Anna Butterworth

The first interstellar dust candidate, dubbed Orion, seen via X-ray fluorescence, which highlights aluminium (red), iron (green) and magnesium (blue).

Seven particles captured by a NASA probe could be the first known samples of interstellar dust to be brought back to Earth.

For the past eight years, a team of researchers — with the help of citizen scientists around the world — have been scanning and analysing the samples brought back to Earth by the Stardust spacecraft.

Interstellar dust flows continuously into the Solar System, but it is extremely sparse, so capturing particles is difficult. Until now, everything scientists knew about such dust has come from analysing spectra of scattered and absorbed light from the interstellar medium. But these observations do not reveal much about the properties of individual particles.

A consortium of 65 scientists studied the microscopic impacts that particles made on Stardust's collectors, which are composed of tiles made from an ultralight material called aerogel. They report their findings today in Science ${ }^{1}$ and in 12 other papers in Meteoritics \& Planetary Science.

\section{First of a kind}

Andrew Westphal, a physicist at the University of California, Berkeley, and lead author of the Science paper, explains that his team faced a chicken-and-egg problem. "Because we had never seen interstellar dust, it was difficult to recognize and differentiate it from interplanetary dust particles," he says. "What we did know were the expected trajectories of the particles as they propagate through the heliosphere," adds Westphal, referring to the vast region of space that covers the Solar System in which the solar wind has influence.

In other words, researchers were looking for dust grains coming from a specific direction in the sky. Westphal says the situation is analogous to driving down a highway in a snowstorm: snowflakes hit the windshield more than they hit the side windows. Similarly, as 
the Solar System orbits the Galaxy, we see dust coming from one particular direction in the sky because of our motion with respect to the interstellar medium.

Of the seven candidate interstellar particles, three — relatively large, at around two microns each — were found on the aerogel tiles of Stardust's collector. The other four particles, much smaller at just a few tenths of a micron across, were found in aluminium foils located between the tiles. Just over half of Stardust's aerogel tiles have been scanned to date, but Westphal does not expect to find more than a dozen particles in the entire collection.

Two of the candidates were revealed at a meeting in 2010 (see 'Volunteer army catches interstellar dust grains'), but the team did not publish their analysis of those paricles — plus five more - until now.

Don Brownlee, an astronomer at the University of Washington in Seattle and Stardust's principal investigator, says that he and his colleagues were quite pleased with the results. "We did not know how successful the collection would be, either in the aerogel tiles or aluminium foils, because no one had ever captured high-speed interstellar material before."

The analyses show that the seven candidate particles are all different from each other in both structure and composition. "This is quite exciting," says Westphal, "because it's telling us this is a much richer and diverse collection of material than we originally expected."

\section{Dust lottery}

Adolf Witt, an astronomer at the University of Toledo in Ohio, considers this a possible breakthrough development: "These are indeed intriguing results that have been awaited by the interstellar-dust community for a long time."

Priscilla Frisch, an astronomer at the University of Chicago in Illinois, agrees. "Dust-grain models will need to be revised to take into account these results," she says. "Interstellar dust particles are a cosmic laboratory for studying the physics of microscopic surfaces and Stardust's results provide a more realistic basis for evaluating the way the grains are charged and the scattering of radiation due to them."

Crucial to the project were the 30,714 volunteers participating in Stardust@home, one of the first online citizen-science initiatives. They collectively carried out more than 100 million image searches looking for tiny interstellar dust impacts on magnified fields of the aerogel.

Naomi Wordsworth from Buckinghamshire, UK, saw the project advertised on the BBC and "thought it sounded fabulous to participate in a real science project open to anyone who wanted to use their skills to help". In 2010, four years after becoming a 'duster', she received an email from Westphal telling her that she had discovered one of the interstellar dust candidates. "It felt like winning the lottery," says Wordsworth. As a reward for her discovery, she was allowed to choose a name for the particle - Hylabrook, after her family's home.

To confirm these preliminary results, the next crucial step will be to measure the relative oxygen isotope abundances in the particles through mass spectroscopy. The team has the necessary instruments, Westphal says, but the particles are still embedded in the aerogel and they are very difficult to handle. "We have such a small collection that we want to be absolutely certain the particles will not be lost. The plan is to spend the next three years, approximately, refining our techniques."

Nature I doi:10.1038/nature.2014.15720

\section{References}

1. Westphal, A. J. et al. Science 345, 786-791 (2014). 